\title{
2015: an eventful year for the Metabolomics Society
}

\author{
Ute Roessner $^{1} \cdot$ Dan Bearden ${ }^{2}$
}

Published online: 6 October 2015

(c) Springer Science+Business Media New York 2015

The Metabolomics Society again has experienced a year full of exciting events and developments. The activity of the Society reflects the growth of the metabolomics sciences with many more researchers in academic, government and industry institutions using and adopting metabolomics in their research in the biological sciences. The Board of Directors of the Society has been working together with the international metabolomics community to expand into new scientific niches across global borders. In the following we aim to summarise important highlights of events and developments within the Metabolomics Society and community over the last year.

\section{Annual meeting and other conferences}

The largest event this year was the annual international meeting in June/July in San Francisco organised and chaired by Prof Oliver Fiehn. The 11th Annual Meeting was the largest conference the society has ever held with nearly 1000 registrants. A detailed summary of the proceedings in San Francisco can be found here http://link. springer.com/article/10.1007/s11306-015-0846-z. One of the most exciting highlights for our early career members of the society was the introduction of additional competitive travel awards for Early-career Members (EMN). In

Ute Roessner

u.roessner@unimelb.edu.au

1 School of BioSciences, The University of Melbourne, Parkville, VIC, Australia

2 National Institute of Standards and Technology, Chemical Sciences Division, Hollings Marine Laboratory, Charleston, SC 29412, USA previous years, the Society has awarded up to ten awards for best posters and presentations to student members only; however, in 2015 the Society also awarded ten additional competitive travel awards for EMN members who are within 5 years post-PhD award. This allowed many more junior researchers to attend the meeting. We also want to acknowledge our partners who also have provided additional student and EMN awards including NIH, the Noble Foundation, UC Davis, RTI International, Sciex and Agilent Technology. We are now looking forward to similar success for our 12th Annual Meeting, which will be held in Dublin, Ireland in June 27-30, 2016 organised and chaired by Prof Lorraine Brennan and Dr Afric O'Sullivan (www. metabolomics2016.org).

In 2015 the Society also sponsored and contributed to a number of regional conferences and workshops. Examples include student travel award support for the Practical Applications of NMR in Industry Conference (PANIC) meeting in San Diego, student travel award support for the Southeast Center for Integrated Metabolomics (SECIM) workshop in Gainesville, Florida, student travel support for the 9th French Metabolomics and Fluxomics (RFMF) meeting in Lille, student travel award support for the 25th Australian and New Zealand Society for Mass Spectrometry, 6th Asia Oceania Mass Spectrometry Conference (AOMSC6/ANZSMS25) in Brisbane, along with major support for the keynote speaker, Prof Lloyd Sumner.

\section{Honorary fellow election}

Every year the Society selects two outstanding scientists for the most prestigious award in the field of metabolomics, the Metabolomics Society Lifetime Honorary Fellowship. In 2015, Prof Robert Hall and Prof Mark Viant were 
elected for their continued commitments to the Society and also for their long-standing contributions to the metabolomics sciences. More information of the two well-known leaders can be found here http://link.springer.com/article/ 10.1007/s11306-015-0820-9.

\section{Early career members network activities}

The most energetic group of individuals in the Society may be the members of the Early-Career Members Network (EMN)! For a glimpse into their many and varied efforts in the last year, please see http://link.springer.com/article/10. 1007/s11306-015-0779-6

\section{Committees and task group activities}

To serve the needs of the Society and community, the Board of Directors have formed a number of standing Committees and functional Task Groups. Committees such as the Conference and Training Committee or the Publication Committee are compromised of elected Board members while our Task Groups include Board members and volunteer Society members willing to participate in community-based activities and contribute to developments on hot topics in the metabolomics sciences. For example, the Data Standard Task Group aims to develop and establish scientific standards for metabolomics data to be adopted by the scientific community (http://link. springer.com/article/10.1007/s11306-015-0821-8). The Metabolite Identification Task Group (http://link.springer. com/article/10.1007/s11306-014-0656-8) works on what may be the most important issue in the field: increasing the number of metabolites identified and raising the confidence levels of identification in metabolomics analyses. The Industry Engagement Task Group establishes and maintains corporate relationships with our Industry partners, an important task to ensure continuous support from our sponsors allowing to keep the costs of the annual meetings low. The Data Quality Task Group (http://link.springer. com/article/10.1007/s11306-014-0679-1) develops QA/QC vocabulary, support of robust measurement procedures and promotes those through educational efforts.

In 2015 the Society has formed a few new Task Groups addressing important issues arising in the community. In San Francisco we established the International Affiliation Task Group, bringing together representatives from existing International Affiliates but also from new regions, which are interested in establishing regional networks, societies or working groups. The aim of this group is to share ideas and experiences, discuss burning regional issues and to learn from each other both scientifically while supporting development of regional metabolomics-related organisations. In addition, the Model Organism Metabolomes Task Group chaired by Prof Viant was founded with the aim to share, discuss and coordinate analytical and bioinformatics strategies to progress the annotation and identification of model organism metabolomes. The new Computational Mass Spectrometry Task Group (CompMS, http://compMS.org/) will build a computational mass spectrometry community to foster computational method developments on signal processing, feature alignment, grouping, metabolite identification and network reconstruction. One major aim of this Task Group is to learn from other 'omics and use synergies between proteomics, metabolomics and other MS-driven fields. At the conference in San Francisco a Task Group for Pharmacometabolomics was founded and will be chaired by Prof Rima Kaddurah-Daouk bringing together many elements of the clinical and pharmaceutical metabolomics communities.

We invite members to contact the Chairs of the Task Groups (see www.metabolomicssociety.org) if they are interested in participating. We always look for motivated Task Group members who bring new ideas and enthusiasm with them.

\section{Establishment of regional chapters}

In 2015 the Board of Directors also had many discussions to develop more formal relationships with regional metabolomics communities and societies than we currently have with our Affiliates through the establishment of Society Chapters. We are excited about these opportunities to work closely with regional initiatives and therefore further strengthen and expand the Metabolomics Society. Initial discussions are ongoing with leaders of the North American metabolomics community and we are hopeful that 2015 will be the year the first Society Chapter is founded. This will lead to many more discussions with other regional networks in the near future and we invite leaders of those initiatives to contact us to start the discussions.

\section{Publication awards}

A highlight of the 2015 annual meeting was the presentation of the 2015 Publication Awards. These two awards recognize the Best paper based on the last 3 years of citations and the Highest Download paper for the total number of electronic downloads over the previous 3 years in the official journal of the Society Metabolomics. For details about the winners, please see http://link.springer. com/article/10.1007/s11306-015-0845-0. 


\section{Looking forward}

The year 2015 has been a monumental year for the Metabolomics Society in numerous ways, some of which have been highlighted here. As the scientific discipline of metabolomics expands in scope and influence, we hope that you can agree that the future is bright. This year represents the second full year of a critical business relationship with
A-S-K Associates, Inc., whose administrative and coordination efforts have given the Board and the Society the capability of doing much, much more than would be imaginable without their steady hand in all things administrative. The Board and Officers are thankful to Sherie Howell and her staff at A-S-K Associates for their 'all in' approach to the Society's welfare and future. Hold on to your hats for 2016 ! 